\title{
Determining the performance of a Diffuser Augmented Wind Turbine using a combined CFD/BEM method
}

\author{
Joss E. Kesby ${ }^{*}$, David R. Bradney, and Philip D. Clausen \\ School of Engineering, Faculty of Engineering and the Built Environment, University of Newcastle, Newcastle, NSW, Australia
}

Received: 28 February 2017 / Received in final form: 6 July 2017 / Accepted: 27 July 2017

\begin{abstract}
Traditionally, the optimisation of a Diffuser Augmented Wind Turbine has focused on maximising power output. However, due to the often less than ideal location of small-scale turbines, cut-in speed and starting time are of equal importance in maximising Annual Energy Production, which is the ultimate goal of any wind turbine design. This paper proposes a method of determining power output, cut-in speed and starting time using a combination of Computational Fluid Dynamics and Blade Element Momentum theory. The proposed method has been validated against published experimental data.
\end{abstract}

\section{Introduction}

The wind conditions found on a typical small wind turbine site are well suited for a Diffuser Augmented Wind Turbine (DAWT). DAWTs have been found to not be adversely affected by turbulent conditions $[1,2]$, and allow a generator to produce power at low wind speeds [3,4]. In combination with rapidly advancing manufacturing techniques, such as 3D printing, the levelised cost of energy of a small-scale wind turbine can be improved, making them more attractive for home or community use either as a standalone energy production system or in a hybrid configuration with solar photovoltaic panels.

The work presented in this paper is part of an ongoing project aimed at producing a DAWT designed to maximise the Annual Energy Production (AEP) for a typical small-scale wind turbine site. Historically, the optimisation of a DAWT has focused primarily on the peak power output of the device. However, due to the lower average wind speed and higher variability of wind on a typical small wind turbine site, three additional performance characteristics have been identified as being important for high AEP: the cut-in speed, starting time and cut-out speed of the turbine. The cut-out speed is dependent on the chosen generator and the strength of the blades, the optimisation of which is deemed to be beyond the scope of this paper. To date, research into optimising the starting time and cut-in speed for a DAWT has been very limited, although similar research for a small-scale horizontal-axis wind turbine has indicated that the parameters are conflicting and an optimisation process will result in a Pareto Front of optimal solutions [5].

This paper will present the simulation methodology of calculating the starting performance and power output of a DAWT and will validate the methodology using data obtained from published experimental and "real world" testing.

\section{Methodology}

\subsection{Power output}

To calculate the power output of a DAWT, a methodology has been developed utilising airflow predictions from Computational Fluid Dynamics (CFD) in combination with power output calculations from Blade Element Momentum (BEM) theory and as such has been designated the combined CFD/BEM method. The method determines the velocity augmentation factor, $\gamma$, through the use of CFD. $\gamma$ is defined as the ratio of the velocity at the blade plane for an empty diffuser to the freestream velocity, a ratio that varies with blade plane radius. The term "empty diffuser" refers to a diffuser with the generator hub modelled but with no blades present. $\gamma$ is determined for a low freestream velocity - the "baseline case" - to allow its use in starting performance calculations. The effective exit area for a diffuser, defined as the cross-sectional area of the non-stalled airflow at the exit of the diffuser, can be calculated using (1):

$$
A_{3 e f f}=\frac{V_{2 a r} A_{2}}{V_{3 m a}},
$$

\footnotetext{
* e-mail: joss.kesby@uon.edu.au
} 
where $V_{2 a r}$ is the area averaged velocity at the blade plane, $A_{2}$ is the throat area of the diffuser and $V_{3 m a}$ is the mass flow averaged velocity at the diffuser's exit. The blade plane is defined as the entire area from the centre body to the diffuser's inner surface at the axial location of the blades and so (1) is essentially the mass continuity equation for incompressible flow. To account for the gap between the blade tip and the inner surface of the diffuser in the BEM calculations, the tip gap is modelled in CFD and the areaaveraged velocity at the diffuser's exit, $V_{3 a r}$, is calculated. Equation (1) can then be updated to:

$$
A_{3 e f f}=\frac{V_{3 a r} A_{3}}{V_{3 m a}},
$$

where $A_{3}$ is the diffuser exit area. The baseline effective exit area, $A_{3 i}$, is calculated using (2) with the velocities obtained from CFD for the baseline freestream velocity.

The diffuser's performance is assessed under a range of freestream velocities in combination with swirling flow and thrust provided by an actuator disc at the blade plane. A relationship between the velocity at the blade plane $\left(V_{2 a r}\right)$ and the mass flow averaged velocity at the exit $\left(V_{3 m a}\right)$ as well as the area averaged velocity at the exit $\left(V_{3 a r}\right)$ is then established for the diffuser. This information is passed to the BEM program, which utilises the baseline velocity augmentation factor, $\gamma_{i}$, to calculate the axial and tangential flow velocity produced by the blades. The value of $\gamma$ is then adjusted based on the equation:

$$
\gamma_{a d j}=\frac{V_{3 m a}}{\beta_{e f f} V_{0} \gamma_{i}(i-a)},
$$

where $V_{0}$ is the freestream velocity, $\gamma_{i}$ is the baseline velocity augmentation factor, $a$ is the axial induction of the airflow and $\beta_{\text {eff }}$ is the increase in the effective exit area over the baseline case given by:

$$
\beta_{\text {eff }}=\frac{A_{2} A_{3 i}}{A_{3 e f f}^{2}} .
$$

The BEM process is then repeated until $\gamma_{a d j}$ converges. Further information on the combined CFD/BEM method can be found in [6].

Experimental studies have shown that blade tip loss can be partially suppressed in a DAWT through the interaction of tip vortices with the inner surface of the diffuser [7]. The level of suppression is dependent on several factors including the proximity of the blade tips to the diffuser surface, the flow velocity, blade tip geometry and tip speed ratio (the latter defined as the ratio of the tangential velocity of the blade tip to the upstream wind velocity).

The classical Prandtl tip loss model has been modified for use with DAWTs by Vaz and Wood [8]. The method detailed by Vaz and Wood uses the standard tip loss equation:

$$
F_{t i p}=\frac{2}{\pi} \cos ^{-1} e^{-f_{t i p}}
$$

but modifies $f_{\text {tip }}$ to account for the increased velocity at the blade element, giving:

$$
f_{\text {tip }}=\frac{B V_{\text {rel }}(R-r)}{2 r \gamma(1-\alpha) V_{0}},
$$

where $B$ is the number of blades, $V_{\text {rel }}$ is the relative velocity at the blade element, $R$ is the total blade length and $r$ is the radial distance to the blade element.

The hub loss model uses a similar equation to (5), with $f_{\text {tip }}$ replaced by $f_{h u b}$, with the latter given by:

$$
f_{h u b}=\frac{B V_{r e l}\left(r-R_{h u b}\right)}{2 r \gamma(1-\alpha) V_{0}},
$$

where $R_{h u b}$ is the hub radius of the turbine. Tip and hub loss has been implemented in the BEM program written for the combined CFD/BEM method, each of which can be toggled on or off by the user.

\subsection{Cut-in speed and starting time}

The calculation of the AEP of a DAWT requires an estimation of the cut-in speed and starting time. A starting code has been developed based on the starting performance concepts as detailed by Wood [9]. The code uses an ordinary differential equation solver to calculate the rate of change of angular velocity of the turbine given by the equation:

$$
\frac{d \Omega}{\mathrm{d} t}=\frac{\left(Q-Q_{r}\right)}{J},
$$

where $Q$ is the aerodynamic torque produced by the turbine, $Q_{r}$ is the resistive torque of the generator and $J$ is the turbine's inertia - a combination of the inertia of the blades and the generator inertia. For a small wind turbine a critical parameter of starting is that of the resistive torque of the generator - the torque required to be produced aerodynamically by the blades before the generator will begin to rotate. A small wind turbine often produces very little torque under starting conditions and a small change in generator resistive torque can have a large impact on the turbine's starting performance.

The starting performance of a turbine is typified by two phases: a period where the turbine first begins rotating where its angular velocity increases gradually in a linear manner (Phase 1), and Phase 2 where the angular velocity increases at a much higher rate. Phase 1 has a linear characteristic due to the blade operating under high angles of attack with a corresponding low lift and high drag, resulting in a low rate of change of angular velocity. As the angular velocity of the blade increases, the angle of attack of the blade begins to approach its optimum, producing more torque and resulting in Phase 2 of the turbine's starting performance.

Under high angles of attack, the lift and drag data of an aerofoil can be approximated by the experimentally derived lift and drag equations of a flat plate. The starting code detailed in [9] utilises flat plate lift/drag polar data for high angles of attack (Phase 1) and aerofoil polar data for 
angles of attack within the aerofoil's operating range (Phase 2). The transition from flat plate to aerofoil polar data occurs at a specified rotor speed, resulting in the lift and drag of the blade being wholly calculated from either flat plate or aerofoil data. The current starting code modifies this to allow blade element-wise transition, allowing each element's lift and drag to be calculated from either aerofoil or flat plate polar data. The transition from one to the other is determined as the intersection point of the aerofoil and the flat plate lift curves. A detailed description of the starting code for use in open-bladed turbines can be found in [10].

The starting code has been adapted for use with a DAWT through the implementation of the augmentation factor to give the velocity profile at the blade plane. The augmentation factor is required to be calculated at a velocity representative of the starting speed and for the current research, this velocity has been determined to be $2 \mathrm{~m} / \mathrm{s}$. Consequently, this is the wind profile that the blades will see from stationary to starting when axial induction is assumed to be negligible. Under this assumption, the blades are deemed to have no influence on the incoming airflow, an assumption which may be valid for rotors with low solidity but may not necessarily be so for high-solidity turbines. The current starting code does not account for solidity effects and further research may be required in this area.

The current starting code uses an iterative, trial-anderror approach to estimate the cut-in speed. An initial velocity is nominated at which the blades will start, $8 \mathrm{~m} / \mathrm{s}$ for the current research, which is then reduced in $0.1 \mathrm{~m} / \mathrm{s}$ increments until the program calculates that the turbine will no longer achieve a successful start, i.e. the rotor Revolutions Per Minute (RPM) at which power production commences is not achieved in the nominated starting time, at which point the program designates the previous velocity as the cut-in speed.

\section{Results}

\subsection{Velocity augmentation}

The velocity augmentation of the empty diffuser is integral to the combined CFD/BEM method for predicting both power output and starting performance. van Dorst provides experimental results for the relative velocity profile $70 \mathrm{~mm}$ behind the blade plane for three unspecified wind tunnel inlet velocities[11]. Figure 1 shows the experimental velocity profiles alongside the relative velocity profile calculated at the same location using CFD for a wind tunnel inlet velocity of $5 \mathrm{~m} / \mathrm{s}$. More detail on the CFD process used for the current research can be found in $[6]$.

The results presented in Figure 1 show that the velocity profile obtained from CFD gives a good estimation of the experimental results, with the largest discrepancy being approximately $6.5 \%$ at a radius of $0.7 \mathrm{~m}$ for Experiment 1 . The baseline augmentation factor used in the current research is for a freestream velocity of $2 \mathrm{~m} / \mathrm{s}$ to accommodate the calculation of starting performance. The effective exit area of the diffuser for the baseline case is $2.45 \mathrm{~m}^{2}$.

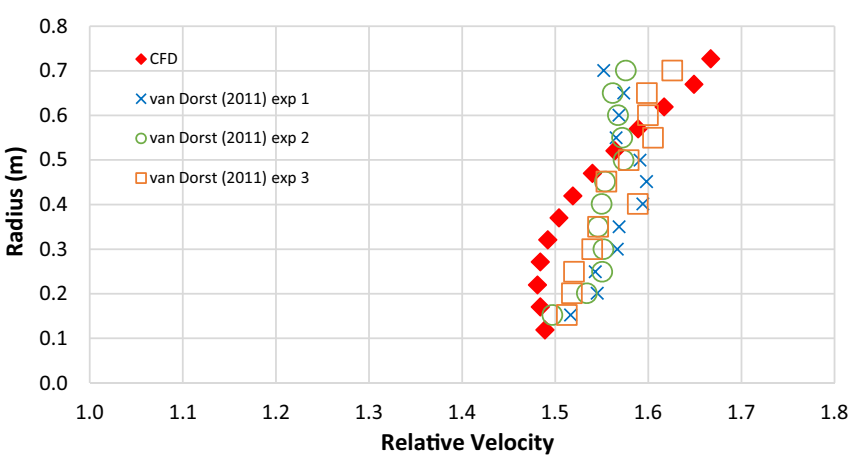

Fig. 1. Experimental and calculated relative velocity profiles for an empty diffuser.

\subsection{Power output}

The combined CFD/BEM method has been validated against published data [11], where a turbine blade profile was optimised for a specific diffuser. van Dorst has presented the experimental power output measured from wind tunnel testing for three different blade profiles:

- the blade originally manufactured for the diffuser (the Original blade);

- an optimised blade for the diffuser that van Dorst developed which had a chord length of up to $230 \mathrm{~mm}$ near the hub (the Optimal blade);

- a modification of the Optimal blade that had a maximum chord length constraint of $130 \mathrm{~mm}$ imposed by the manufacturer. A section of the blade nearest the hub had its chord length reduced to accommodate this constraint, giving the blade a more linear appearance and hence being designated the Linear blade.

The power output was measured for each blade configuration for four inlet velocities: $5 \mathrm{~m} / \mathrm{s}, 6 \mathrm{~m} / \mathrm{s}, 7 \mathrm{~m} / \mathrm{s}$ and $8 \mathrm{~m} / \mathrm{s}$. van Dorst allowed for wind tunnel blockage effects and calculated that the actual wind tunnel inlet velocities were $4.9 \mathrm{~m} / \mathrm{s}, 5.9 \mathrm{~m} / \mathrm{s}, 6.8 \mathrm{~m} / \mathrm{s}$ and $7.8 \mathrm{~m} / \mathrm{s}$. The corrected velocities were used for the BEM calculations although, for clarity, the ongoing discussion will refer to the intended velocities without blockage effects. Figure 2 shows the experimental power output for the three blade geometries plotted with the predicted power outputs both with and without tip loss (hub loss was included for both sets of results).

Figure 2 shows that the predicted results are in good agreement with the experimental data and, in general, the predicted results with and without tip loss encompass the experimental data. The calculated results without tip loss over predicted the experimental results for all geometries and wind tunnel velocities, with the largest error being $24 \%$ for the Original blade at $5 \mathrm{~m} / \mathrm{s}$. The CFD/BEM Method with tip loss provided a reasonable approximation of the experimental power output. The largest discrepancy was $15.4 \%$ for the Optimal blade at $5 \mathrm{~m} / \mathrm{s}$ but the majority of results were within $10 \%$ of experimental values. While the control system used for the experimental turbine may account for some of the differences between experimental and predicted power output, further work on an improved tip loss model may improve this correlation. Through wind 

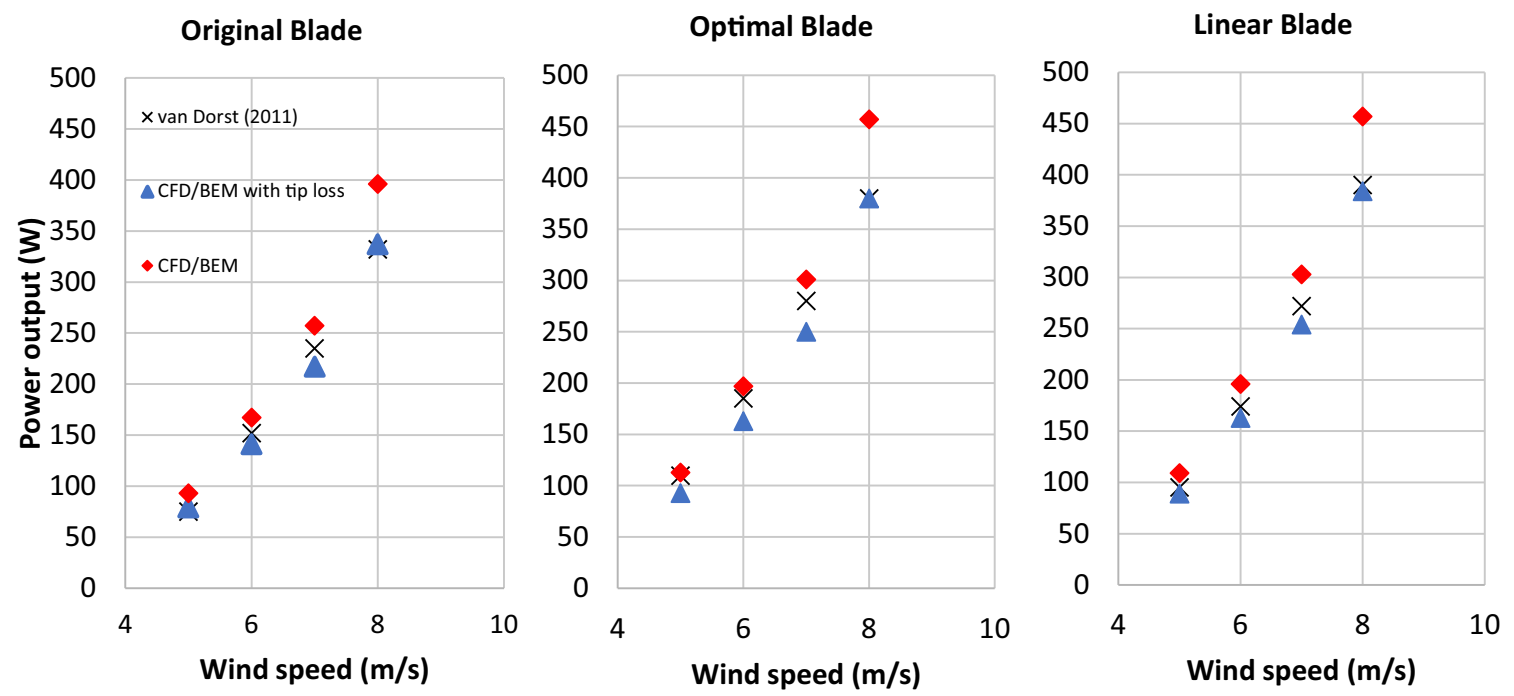

Fig. 2. Experimental and predicted power output for the three blade geometries.
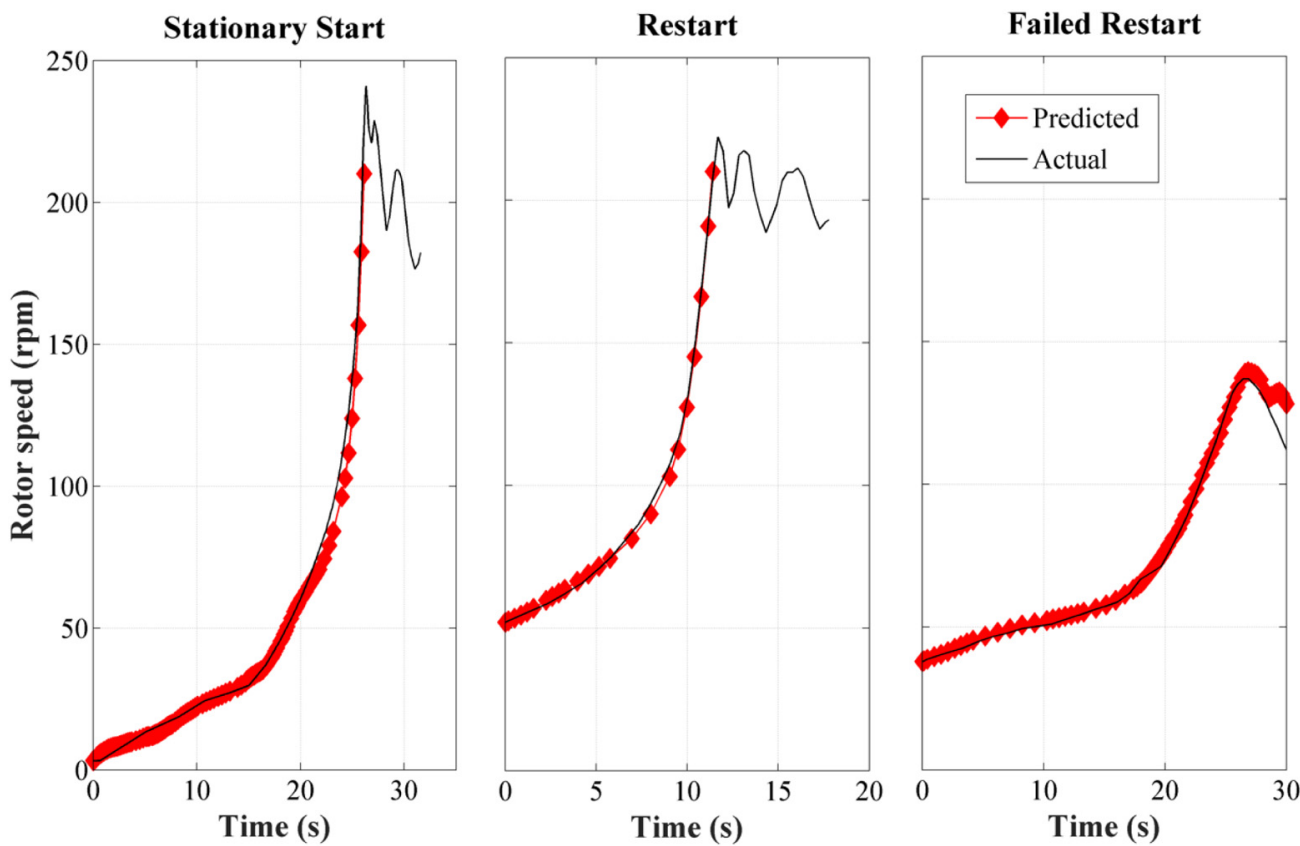

Fig. 3. Calculated and experimental starting performance of an open-bladed wind turbine for three different starting regimes. Power production commences at a rotor speed of $175 \mathrm{RPM}$.

tunnel testing, further validation of the combined $\mathrm{CFD} /$ BEM method will be undertaken at the University of Newcastle.

\subsection{Cut-in speed and starting time}

An extensive amount of field data has been collected from a $5 \mathrm{~kW}$ open-bladed wind turbine located at the University of Newcastle. The data includes wind direction, wind speed, turbine rotor RPM, turbine electrical power output and turbine heading, allowing the starting time of the turbine to be determined over a wide range of wind conditions. This has allowed the starting code to be validated against experimental data under a variety of wind regimes. The code developed at the University of Newcastle[10] can account for dynamic conditions such as increasing wind speeds and yaw misalignment. Figure 3 shows the predicted and experimental starting performance of the $5 \mathrm{~kW}$ wind turbine under three different starting regimes: starting from stationary, restarting (when the wind speed has decreased below the level required to maintain power production and then increased again while the blades are still rotating) and a failed restart. The turbine commences power production at a rotor speed of $175 \mathrm{RPM}$.

It can be seen from Figure 3 that the predicted starting performance is in very good agreement with the field data for all three data sets. The starting performance for the current work is being calculated under steady state 
Table 1. Measured and predicted cut-in speeds for the three blade geometries.

\begin{tabular}{llll}
\hline Blade geometry & Measured & Predicted $(0.2 \mathrm{Nm})$ & Predicted $(0.5 \mathrm{Nm})$ \\
\hline Original & $6.2 \mathrm{~m} / \mathrm{s}$ & $3.7 \mathrm{~m} / \mathrm{s}$ & $6.2 \mathrm{~m} / \mathrm{s}$ \\
Optimal & $3.2 \mathrm{~m} / \mathrm{s}$ & $1.8 \mathrm{~m} / \mathrm{s}$ & $2.8 \mathrm{~m} / \mathrm{s}$ \\
Linear & $3.7 \mathrm{~m} / \mathrm{s}$ & $2 \mathrm{~m} / \mathrm{s}$ & $3.1 \mathrm{~m} / \mathrm{s}$ \\
\hline
\end{tabular}

conditions (constant wind speed with little turbulence and no yaw misalignment) and so the correlation of the predicted starting to the experimental starting under the much more complex field conditions is viewed as a very good validation of the starting code.

There is very little published data regarding the starting performance for a DAWT. van Dorst [11] provides experimental cut-in wind speeds for the three tested blades although no mention is made of starting time. A plot of torque against generator power is also provided and a resistive torque for use in the current research has been extrapolated from this data, providing an approximation for use with the developed starting program. The extrapolated resistive torque is approximately $0.2 \mathrm{Nm}$ which is low for a generator of this size $(1.75 \mathrm{~kW})$. In comparison, the original generator in the $5 \mathrm{~kW}$ turbine at the university had a resistive torque of $0.9 \mathrm{Nm}$ and has since been replaced with a generator that has a resistive torque of $1.9 \mathrm{Nm}$. Based on information provided in [11], the generator will begin power production at $300 \mathrm{RPM}$ and the maximum starting time has been nominated as $90 \mathrm{~s}$.

The cut-in speeds for the three blade geometries were estimated for two resistive torques: $0.2 \mathrm{Nm}$ and $0.5 \mathrm{Nm}$. The former was the aforementioned extrapolation of experimental data and the latter was calculated by adjusting the resistive torque until the old blade started at the cut-in speed measured by van Dorst $(6.2 \mathrm{~m} / \mathrm{s})$; this resistive torque was then used to predict the cut-in speed for the other two blades. Table 1 lists the measured and predicted cut-in speeds for the three blade geometries.

As can be seen from Table 1, both sets of predicted results underestimate the cut-in speeds of the three blade geometries except, of course, for the original blades with a resistive torque of $0.5 \mathrm{Nm}$. When using a resistive torque of $0.2 \mathrm{Nm}$, the cut-in speeds were under predicted by up to $46 \%$. The results greatly improved when using a resistive torque of $0.5 \mathrm{Nm}$, although the cut-in speeds were still underestimated by up to $17 \%$.

\section{Conclusions}

The work described a simulation methodology, designated the combined CFD/BEM method, to determine the performance of a DAWT. The method calculates both the starting performance and power output and has been validated against experimental data, with further wind tunnel validation to be undertaken in the near future.

The comparison of predicted power output with published experimental data [11] indicates that while the power output of a DAWT can be estimated to a reasonable degree of accuracy (within 15\%), an updated method of calculating tip loss should see an improvement in the accuracy of the predicted results.
While the starting code developed at the University of Newcastle [10] accurately models the starting performance of an open-bladed $5 \mathrm{~kW}$ turbine under a broad range of operating conditions, the code underestimates the cut-in wind speed of a DAWT. Uncertainty around the resistive torque of the experimental generator used in the validation could have had a significant impact on the accuracy of predictions. A further potential source of error is the high solidity of the DAWT's blades in comparison to that of the open turbine, particularly the Optimal and Linear blades. The assumption of no axial induction at the blade plane used in the starting code may be valid for low-solidity turbines but a high solidity will present more of an obstacle to the airflow, potentially reducing the wind speed at the blade plane with a resultant non-negligible axial induction. The local blade solidity for the open-bladed $5 \mathrm{~kW}$ turbine at the University of Newcastle is between 0.01 and 0.2 whereas the solidity for the Original, Optimal and Linear turbines are between 0.03-0.3, $0.06-1.7$ and $0.06-1.2$ respectively. To date, the impact of blade solidity on DAWT starting performance has not been investigated, but may represent a good avenue for future research and could prove beneficial for the optimisation of a small scale DAWT.

\section{References}

1. S.W. Armfield, C.A.J. Fletcher, Comparison of $k-\varepsilon$ and algebraic Reynolds stress models for swirling diffuser flow, Int. J. Numer. Methods Fluids 9, 987 (1989)

2. D.G. Phillips, An Investigation on Diffuser Augmented Wind Turbine Design, PhD in Engineering, Ph.D. Thesis, Department of Mechanical Engineering, The University of Auckland, New Zealand, 2003

3. Y. Ohya, T. Karasudani, A. Sakurai, K. Abe, M. Inoue, Development of a shrouded wind turbine with a flanged diffuser, J. Wind Eng. Ind. Aerodyn. 96, 524 (2008)

4. F. Bet, H. Grassmann, Upgrading conventional wind turbines, Renew. Energy 28, 71 (2003)

5. M. Sessarego, K. Dixon, D. Rival, D. Wood, A hybrid multiobjective evolutionary algorithm for wind-turbine blade optimization, Eng. Optim. 47, 1043 (2015)

6. J.E. Kesby, D.R. Bradney, P.D. Clausen, Determining Diffuser Augmented Wind Turbine performance using a combined CFD/BEM method, J. Phys.: Conf. Ser. 753, 082033 (2016)

7. K. Abe, H. Kihara, A. Sakurai, E. Wada, K. Sato, M. Nishida et al., An experimental study of tip-vortex structures behind a small wind turbine with a flanged diffuser, Wind Struct. Int. J. 9, 413 (2006)

8. J.R.P. Vaz, D.H. Wood, Aerodynamic optimization of the blades of diffuser-augmented wind turbines, Energy Convers. Manag. 123, 35 (2016)

9. D. Wood, Small Wind Turbines (Springer, Berlin, Heidelberg, 2011) 
10. D.R. Bradney, Measured and Predicted Performance of a Small Wind Turbine Operating in Unsteady Flow, Doctoral Dissertation, School of Engineering, University of Newcastle, Australia, 2017
11. F. van Dorst, An Improved Rotor Design for a Diffuser Augmented Wind Turbine, Master's Thesis, Aerospace Engineering, Delft University of Technology, Netherlands, 2011

Cite this article as: Joss E. Kesby, David R. Bradney, Philip D. Clausen, Determining the performance of a Diffuser Augmented Wind Turbine using a combined CFD/BEM method, Renew. Energy Environ. Sustain. 2, 22 (2017) 